\title{
Effectiveness of In Vitro Primary Coronal Caries Prevention with Silver Diamine Fluoride- chemical vs biofilm models
}

Short title: Primary Coronal Caries Prevention with Silver Diamine Fluoride

Parand Sorkhdini ${ }^{1}$, Richard L. Gregory ${ }^{2}$, Yasmi O. Crystal ${ }^{3}$, Qing Tang ${ }^{4}$, Frank Lippert ${ }^{1}$

1- Department of Cariology, Operative Dentistry and Dental Public Health, Indiana University School of Dentistry, Indianapolis, IN, USA; e-mail: Parsorkh@iu.edu

2- Department of Biomedical Sciences and Comprehensive Care, Indiana University School of Dentistry, Indianapolis, IN, USA; e-mail: rgregory@iu.edu

3- Department of Pediatric Dentistry, NYU College of Dentistry; New York, USA; e-mail: Yoc1@nyu.edu

4- Department of Biostatistics, Indiana University School of Medicine, Indianapolis, IN, USA; email: qingtang@iu.edu

*Corresponding author:

Frank Lippert

Indiana University School of Dentistry

Department of Cariology, Operative Dentistry and Dental Public Health

Oral Health Research Institute

415 Lansing Street, Indianapolis, IN 46202 (USA)

Tel. +1 317274 3983, Fax +1 317274 5425, E-Mail flippert@iu.edu

\begin{abstract}
Objectives: The main goal of this study was to investigate the effectiveness of SDF and its individual components, silver $\left(\mathrm{Ag}^{+}\right)$and fluoride $\left(\mathrm{F}^{-}\right)$ions, in preventing enamel demineralization using biofilm and chemical models.

Methodes: Polished human enamel specimens were assigned to five treatment groups ( $\mathrm{n}=18$ per group): SDF (38\%); SDF followed by application of a saturated solution of potassium iodide (SDF+KI); silver nitrate $\left(\mathrm{AgNO}_{3}\right.$; silver control, 253,900 ppm Ag$)$; potassium fluoride (KF; fluoride control, 44,800 ppm
\end{abstract}

This is the author's manuscript of the article published in final edited form as:

Sorkhdini, P., Gregory, R. L., Crystal, Y. O., Tang, Q., \& Lippert, F. (2020). Effectiveness of in vitro primary coronal caries prevention with silver diamine fluoride - Chemical vs biofilm models. Journal of Dentistry, 99, 103418. https://doi.org/10.1016/j.jdent.2020.103418 
F); deionized water (DIW). Treatments were applied once to sound enamel. In the biofilm model, specimens were demineralized by aerobic overnight incubation using cariogenic bacteria isolated from human saliva in brain heart infusion supplemented with $0.2 \%$ sucrose for three days. In the chemical model, enamel specimens were immersed in a demineralizing solution containing $0.1 \mathrm{M}$ lactic acid, $4.1 \mathrm{mM} \mathrm{CaCl}, 8.0$ $\mathrm{mM} \mathrm{KH}_{2} \mathrm{PO}_{4}, 0.2 \%$ Carbopol 907, $\mathrm{pH}$ adjusted to 5.0 for five days. Vickers surface microhardness was used to determine the extent of enamel demineralization. Data were analyzed using one-way ANOVA.

Results: In the chemical model, there was no statistically significant difference between SDF and SDF+KI in preventing coronal caries $(\mathrm{p}<0.0001)$. In the biofilm model, SDF+KI was significantly less effective in preventing demineralization than SDF $(\mathrm{p}<0.0001)$. In both models, SDF and SDF+KI were superior in their ability to prevent caries lesion formation than $\mathrm{AgNO}_{3}$ and DIW.

Conclusion: KI application after SDF treatment appears to impair SDF's ability to prevent biofilm-mediated but not chemically induced demineralization.

Clinical significance: SDF may be a viable option in preventing primary coronal caries.

Keywords: Silver Diamine Fluoride, Dark staining, Demineralization, Biofilm, Caries Prevention, Fluoride 


\section{Introduction}

Over the last 20 years, the high prevalence of dental caries and the increasing cost of healthcare pose a significant public health problem all over the world. In this addition, treatment of dental caries in young, fearful, non-cooperative children or those with limited access to dental care or financial limitations can be challenging as untreated dental caries in children can cause pain, infections, and costly emergency room visits and/or hospitalizations. Moreover, current methods of early caries preventive treatment do not seem to successfully inhibit caries development [1,2]. The current trend to manage the dental caries process aims to utilize minimally invasive, tissue-preserving, affordable and safe approaches, while efforts to create more effective anti-caries agents are still ongoing and desperately needed. Silver fluoride, or in stabilized forms such as silver diamine fluoride (SDF), has been used in Japan as early as the 1970s for both the treatment of dentinal hypersensitivity and dental caries and it has been rapidly implemented by dentists in the United States since 2015 [3]. Silver diamine fluoride $\left(\mathrm{Ag}\left(\mathrm{NH}_{3}\right)_{2} \mathrm{~F}\right)$ is a colorless aqueous solution which contains both silver $\left(\mathrm{Ag}^{+}\right)$and fluoride $\left(\mathrm{F}^{-}\right)$ions. Silver is an antimicrobial agent which attacks cariogenic bacteria, promotes resistance to biofilm (re-)formation, while fluoride promotes remineralization of the tooth. SDF is a safe, minimally invasive approach which is effective and affordable and might be helpful to those with special care needs and for lower income groups [4,5].

SDF has proven clinical efficacy for caries arrest on dentinal caries lesions in primary teeth [6] and limited evidence indicates its effectiveness on caries arrest on permanent teeth [7] and there is also limited clinical evidence of SDF preventing new lesions formation. In vitro studies have shown that SDF prevents the formation of cariogenic biofilms [8] including mono-species biofilms of Streptococcus mutans and Actinomyces naeslundii. SDF was also shown to prevent dentin demineralization [9]. SDF is currently being used in the arrestment of active dentin carious lesions. The major drawback associated with SDF is the permanent black staining that results precipitation of silver ions on demineralized enamel. These silver ions precipitate as $\mathrm{Ag}_{2} \mathrm{~S}$ and react with organic material, leaving a black staining on the teeth which can be obvious depending on the location of the dental caries lesion [10]. Therefore, a substantial barrier to widespread use of SDF is the patient/parental unwillingness to accept a permanent black staining [10,11]. Based on Knight et al., a possible solution to minimize the staining issue is to apply saturated solution KI immediately after SDF application to bind free silver ions from SDF [12]. KI reacts with free silver ions and forms a yellow precipitate of AgI, which is insoluble in water and prevents the black staining caused by SDF. However, no adequate in vitro data on the anti-caries efficacy of SDF and SDF+KI as a preventive agent in enamel could be retrieved. Moreover, the ability of SDF to prevent the demineralization of sound dental enamel; i.e., primary coronal caries prevention, is yet to be investigated. Although permanent and primary enamel have some inherent differences (mineral composition, enamel rod density and overall thickness), the mechanisms of caries progression and remineralization are reported to be similar; therefore 
for the purpose of this study, we chose to use permanent enamel $[13,15]$. The present laboratory study aimed to: 1) evaluate the efficacy of SDF for caries prevention in enamel, 2) evaluate if applying KI after SDF affects its anti-caries efficacy while simultaneously retarding staining issues, and 3) compare chemical vs. biofilm models in inducing demineralization to study the differential efficacy of caries preventive agents. We hypothesized that a) SDF is an effective anti-caries agent in the inhibition of enamel demineralization, b) KI application immediately after SDF treatment can significantly reduce staining caused by SDF alone while not affecting SDF anti-caries efficacy, and c) SDF may be comparatively more effective in inhibiting demineralization in a biofilm model than in a chemical model.

\section{Materials and Methods}

\subsection{Study Design}

The study was determined to be exempt from IRB oversight IRB \#: NS0911-07. The schematic of the experimental procedures in this study is shown in Fig 1. Briefly, 180 polished human permanent enamel specimens were assigned to five treatment groups after color and surface microhardness assessments: SDF, $\mathrm{SDF}+\mathrm{KI}, \mathrm{AgNO}_{3}, \mathrm{KF}$ and DIW. Color assessment immediately after treatment application was performed only in the chemical model. Specimens were then demineralized using using two different demineralization models - chemical and biofilm for five and three days, respectively. The biofilm isolated from the enamel blocks in the biofilm model was analyzed for Colony-Forming Units (CFU). All enamel samples were analyzed for changes in color and surface microhardness and using transverse microradiography (TMR) to determine integrated mineral loss and lesion depth.

\subsection{Specimen selection and preparation}

One hundred and eighty sound extracted human permanent teeth predominantly molars and premolars (anonymous donations from dental clinics) were used as specimens. Only buccal and/or lingual surfaces with no wear defects, fracture lines, or cracks were included in this study. Tooth crowns were cut into $4 \times 4$ mm specimens using a low-speed saw (Iso Met, Buehler, Lake Bluff, IL, USA). The teeth were stored in deionized water (DIW) containing thymol $(0.1 \% \mathrm{w} / \mathrm{v})$ during the sample preparation process. Specimens were ground and polished to create flat, planar parallel enamel surfaces using a Struers Rotopol 31/Rotoforce 4 polishing unit (Struers Inc., Cleveland, Pa., USA). The enamel specimens were serially ground using 1,200-, 2,400-, and 4,000-grit silicon carbide grinding paper. The specimens were then polished using a $1-\mu \mathrm{m}$ diamond polishing suspension on a polishing cloth until the enamel surface had a minimum of a $3 \times 4 \mathrm{~mm}$ highly polished facet across the specimen. The resulting specimens had a thickness range of 1.7-2.2 mm. (enamel and underlying dentin). The specimens were assessed under a Nikon SMZ 
1500 stereomicroscope at $20 \times$ magnification for cracks, hypomineralized (white spot) areas, or other flaws in the enamel surface that would exclude them from use in the study. An experimental window, measuring approximately $2 \times 4 \mathrm{~mm}$, was created on the human enamel specimens using acid-resistant, colored nail varnish (Sally Hansen Advanced Hard as Nails Nail Polish, USA), leaving sound enamel areas on either side. Specimens were stored at $100 \%$ relative humidity at $4^{\circ} \mathrm{C}$ until further use [16].

\subsection{Pretreatment assessment}

\subsubsection{Sound enamel color assessment}

Color assessments were performed by a single examiner to evaluate color changes among the treatment options. Commision Internationale de l'Eclairage (CIE) $\mathrm{L}^{*}$ values were recorded. Measurements were performed using a spectrophotometer, Minolta Chroma meter CR-241 (Minolta Camera Co., Osaka, Japan) with D65 light against a white background. Calibration of the spectrophotometer was performed using a ceramic tile supplied by the manufacturer. The area of the specimens scored was a 3-mm diameter circle in the center of the enamel surface. All measurements were repeated three times [17].

\subsubsection{Sound enamel surface microhardness}

Specimens were assessed for sound enamel SMH using a microhardness tester (2100 HT; Wilson Instruments, Norwood, MA, USA). Each enamel specimen was secured on a 1-inch square acrylic block with sticky wax and then placed in the center of the hardness tester. Four baseline indentations spaced $100 \mu \mathrm{m}$ apart were placed with a Vickers diamond under a $200 \mathrm{~g}$ load in the center of a flattened, polished sound enamel specimen, each with a dwelling time of $11 \mathrm{~s}$. SMH was determined by measuring the indentation length using dedicated image analysis software (Clemex CMT HD version 6.0.011, Clemex Technologies Inc., Longueuil, Quebec, Canada).

$\mathrm{SMH}_{\text {sound }}$ was derived from the respective indentation lengths and recorded. Only specimens which fulfilled the criteria of $300 \leq \mathrm{SMH}_{\text {sound }} \leq 400$ were acceptable for use in the study and were divided into groups for each treatment and intervention group within each study.

\subsubsection{Specimen stratification}

The enamel specimens were stratified into two study groups with 18 specimens per study group to ensure that there were no significant differences in $\mathrm{SMH}_{\text {sound }}$ between treatment and study groups.

\subsubsection{Biofilm model pretreatment sanitization of the enamel blocks:}

In order to avoid contamination and the growth of environmental microbes, samples were dipped into $70 \%$ alcohol for 2 seconds and air dried for 15 min before applying the treatment solutions. 


\subsection{Treatment groups}

Enamel specimens were randomized into five treatment groups of 36 specimens each: SDF, SDF+KI, $\mathrm{AgNO}_{3}, \mathrm{KF}$ and DIW (placebo groups).

- SDF: 38\% SDF (Advantage Arrest, Elevate Oral Care LLC, Fl, USA) solution; nominally 253,900 ppm $\mathrm{Ag}^{+} ; 44,800 \mathrm{ppm} \mathrm{F}^{-}$

- SDF+KI: SDF application followed by supersaturated KI application (Potassium iodide 39\% w/v solution, 30315, Sigma-Aldrich, St. Louis, Mo, US)

- $\mathrm{AgNO}_{3}$ : silver control; 253,900 ppm Ag (Silver nitrate 31630, Sigma-Aldrich)

- KF: fluoride control; 44,800 $\mathrm{ppm} \mathrm{F}^{-}$(Potassium fluoride 60238, Sigma-Aldrich)

- DIW: negative control

A micro applicator (Regular; Premium Plus International Ltd., Hong Kong, China) was used to apply SDF solution. All other solutions were applied to the specimen's enamel surface with a micro-brush (Premium Plus Regular Tip Micro A microbrush). All solutions were left on the enamel surface undisturbed for 60 min before color assessment. For the SDF+KI group, SDF was applied immediately followed by a saturated KI solution until the creamy yellow solution turned clear, and the reaction products were wiped off using sterile cotton swabs.

\subsection{Post-treatment color assessment}

In the chemical model, color assessments were performed again, after application of the interventions. In the biofilm model, in order to avoid contamination of the specimen with environmental bacteria, color assessments were not performed between baseline and after treatment. $\mathrm{L}^{*}$ was recorded for each specimen and the following variable was calculated: $\Delta \mathrm{L}^{*}=\mathrm{L}^{*}$ post $-\mathrm{L}^{*}$ sound. All measurements were repeated three times.

\subsection{Demineralization using chemical model}

Immediately after color measurements, early caries lesions were created in the specimens utilizing a modified demineralization protocol based on the White (1987) protocol [18]. Artificial lesions were formed in the enamel specimens by a 5-day immersion in a solution containing $0.1 \mathrm{M}$ lactic acid, $4.1 \mathrm{mM} \mathrm{CaCl}_{2} \times$ $2 \mathrm{H}_{2} \mathrm{O}, 8.0 \mathrm{mM} \mathrm{KH}_{2} \mathrm{PO}_{4}$ (all Sigma-Aldrich) and 0.2\% w/v Carbopol 907 (BF Goodrich Co., USA), pH adjusted to 5.0 using $\mathrm{KOH}$, at $37^{\circ} \mathrm{C}$ [18]. The protocol of 5-day immersion was based on pilot data. Demineralization was performed at a ratio of $10 \mathrm{ml}$ of solution per specimen. After lesion creation, specimens were rinsed with DIW and stored at $100 \%$ relative humidity at $4{ }^{\circ} \mathrm{C}$. 


\subsection{Demineralization using biofilm model}

The blocks (not the enamel surface) were disinfected/sanitized using 70\% ethanol wipe, and then they were kept under the UV light for $15 \mathrm{~min}$. After specimen preparation was completed, specimens were mounted on the lid of a six-well plate (Fisher Scientific Co., Silver Spring, Md.) with acrylic cubes. Specimens were demineralized by aerobic incubation in a clinically relevant overnight culture of cariogenic bacteria including S. mutans. An overnight culture of multi-species bacterial mix, which was previously collected from human saliva under IRB approval \#1406440799 based on Ayoub et al. 2019 [19], was mixed with an overnight culture of $S$. mutans strain UA159 (ATCC 700610) in a 10:1 volume ratio. Each specimen was incubated in a six-well tissue culture plate containing the bacterial inoculum for $72 \mathrm{~h}$, aerobically to create caries lesions. This time period was chosen to achieve a similar level of demineralization to the chemical model. The media and the plates were changed daily. The growth media contained Brain Heart Infusion (BHI) supplemented with $0.2 \%$ sucrose. This experiment was repeated three times with six samples per group (totaling $\mathrm{n}=18$ per intervention group).

\subsection{Post-intervention assessment}

\subsubsection{Colony counting}

Isolated biofilm was analyzed for bacterial viability using an established method [20]. For CFU counting, biofilm on the exposed surfaces of the enamel blocks was wiped off with a micro brush. The tip of the micro brush was placed in $1 \mathrm{ml}$ of saline and sonicated. One hundred $\mu 1$ of the biofilm suspension was spread with a sterile glass rod on blood agar plates and incubated for $48 \mathrm{~h}$ in aerobic conditions at $37^{\circ} \mathrm{C}$. Finally, the colonies on the plates were counted to calculate CFU/ml. Again, after lesion creation, enamel specimens were rinsed with DIW, and kept at $100 \%$ relative humidity at $4^{\circ} \mathrm{C}$ until future analysis.

\subsubsection{Post-intervention color assessment}

In both chemical and biofilm models, color assessments were performed after demineralization. L* was recorded for each specimen and the following variable was calculated: $\Delta \mathrm{L}^{*}$ intervention $=\mathrm{L}^{*}$ intervention $_{\text {- }} \mathrm{L}_{\text {sound. }}$. All measurements were repeated three times.

\subsubsection{Surface microhardness change}

After completion of the studies, all specimens were again subjected to surface microhardness measurements as described above. A second set of four indentations was placed on each specimen in close proximity and to the right of the baseline indentations, yielding $\mathrm{SMH}_{\text {post. }}$ The extent of percent change in $\mathrm{SMH}$ for each individual specimen was calculated as follows: \%SMHchange $=100 *\left(\mathrm{SMH}_{\text {sound }}-\mathrm{SMH}_{\text {post }}\right) / \mathrm{SMH}_{\text {sound }}$. 


\subsubsection{Transverse Microradiography}

One section per specimen, approximately $100 \mu \mathrm{m}$ in thickness, was cut from the center of each specimen and across the lesion window and sound enamel areas using a Silverstone-Taylor Hard Tissue Microtome (Scientific Fabrications Laboratories, USA). The sections were placed in the TMR-D system and X-rayed at $45 \mathrm{kV}$ and $45 \mathrm{~mA}$ at a fixed distance for $12 \mathrm{~s}$. An aluminum step wedge was also X-rayed under identical conditions. The digital images were analyzed using the TMR software v.3.0.0.18. A window (approximately $400 \times 400 \mu \mathrm{m}$ ), representative of the entire lesion area and not containing any cracks, debris or other alterations, was selected for analysis. Sound enamel mineral content was assumed to be $85 \% \mathrm{v} / \mathrm{v}$. The following variables were recorded for each specimen/section: $\Delta \mathrm{Z}$ - integrated mineral loss: (product of lesion depth and the mineral loss over that depth), L - lesion depth.

\subsection{Statistical Analysis}

With a sample size of 18 specimens per group in each part of the study, the study has $80 \%$ power to detect a difference of $10 \%$ for $\%$ SMHchange, $15 \%$ for $\Delta \mathrm{Z}$, and $27 \%$ for Land $27 \%$ for CFU. The calculations assume two-sided tests conducted at a 5\% significance level for each type of comparison, with coefficients of variance estimated at 0.1 for \%SMHchange, 0.15 for $\Delta \mathrm{Z}$, and $0.27 \mathrm{~L}$ and 0.27 for CFU.

Separate analyses were performed for biofilm and chemical models. VHN hardness (the percent change in surface microhardness), mineral loss, lesion depth, log-transformed CFU and color changes $\left(\Delta \mathrm{L}^{*}\right)$ were analyzed using one-way ANOVA to examine the effect of treatment types. Experiment units were included in the model as a random effect. All pair-wise comparisons from ANOVA analysis were made using Fisher's Protected Least Significant Differences to control the overall significance level at 5\%. Analyses were performed using SAS version 9.4 (SAS Institute, Inc., Cary, NC).

\section{Results}

\subsection{Microhardness}

The \%SMHchange data for both models are shown in Figs. 2 and 3. In the chemical model, there were no statistically significant differences between SDF and SDF+KI ( $\mathrm{p}=0.0515)$ in preventing enamel demineralization. There were statistically significant differences between SDF and SDF+KI in preventing caries lesion formation compared to $\mathrm{KF}, \mathrm{AgNO}_{3}$ and DIW (all $\mathrm{p}<0.0001$ ). $\mathrm{AgNO}_{3}$ and DIW exhibited a significant reduction in their VHN values compared to KF (both $\mathrm{p}<0.0001$ ). There was no difference between $\mathrm{AgNO}_{3}$ and DIW ( $\mathrm{p}=0.1756$ ). In the biofilm model, there were statistically significant differences in preventing caries lesion formation between SDF and SDF+KI ( $p<0.0001) . \mathrm{SDF}+\mathrm{KI}, \mathrm{AgNO}_{3}$ and DIW exhibited a significant reduction in their VHN values compared to KF (all $p<0.0001$ ). There was no 
difference between SDF and KF ( $\mathrm{p}=0.0690)$. There was also no difference between $\mathrm{AgNO}_{3}$ and DIW $(\mathrm{p}=0.2380)$.

\section{$3.2 T M R$}

The $\Delta \mathrm{Z}$ and $\mathrm{L}$ data for both models and all treatment groups can be found in Table 1. In the chemical model, there was a significant difference between SDF, SDF+KI, and KF in demineralization inhibition compared to $\mathrm{AgNO}_{3}$ and DIW ( $\mathrm{p}<0.0001$ ). In the biofilm model, for both $\Delta \mathrm{Z}$ and $\mathrm{L}$, there were no statistically significant differences between any of the treatment groups ( $\mathrm{p}=0.0750$ and $\mathrm{p}=0.1659$, repectively).

\section{$3.3 \quad C F U$}

$\mathrm{CFU} / \mathrm{ml}$ values for the biofilm model are shown in Fig 4. There was a significant difference between SDF and SDF+KI in inhibiting $S$. mutans and other salivary bacteria compared to $\mathrm{AgNO}_{3}$ and $\mathrm{DIW}(\mathrm{p}<0.0001)$. There were no differences between $\mathrm{KF}, \mathrm{AgNO}_{3}$ and DIW ( $\left.\mathrm{p} \geq 0.07\right)$.

\section{$3.4 \quad$ Color Assessment}

$\Delta \mathrm{L}^{*}$ data for both models and all treatment groups are shown in Fig 5 and $6 . \Delta \mathrm{L}^{*}$ values were evaluated for after treatment change from baseline and post-intervention change from baseline in the chemical model, as well as post-intervention change from baseline in the biofilm model. In both chemical and biofilm models, $\mathrm{L}^{*}$ values from baseline to post intervention demonstrate applying KI after SDF significantly reduced the dark staining caused by SDF $(\mathrm{p}<0.0001)$. Accordingly, SDF+KI groups had significantly higher $\Delta \mathrm{L}^{*}$ values than SDF alone, whereas group SDF and $\mathrm{AgNO}_{3}$ groups presented significantly lower $\Delta \mathrm{L}^{*}$ compared with $\mathrm{SDF}+\mathrm{KI}$ groups.

\section{Discussion}

To the authors' knowledge, this is the first study that investigated the ability of SDF to prevent enamel demineralization while utilizing both chemical and biofilm models. The present research is therefore significant, as understanding the mechanism behind SDF could lead to more widespread use of SDF in primary coronal caries prevention. Appropriate silver $\left(\mathrm{AgNO}_{3}\right)$ and fluoride $(\mathrm{KF})$ controls as well as two models of demineralization, chemical and biofilm, were included to elucidate the mode of action of SDF. Furthermore, KI was investigated as a post-SDF application treatment to mitigate staining associated with SDF.

A distinct difference in the comparative efficacy of SDF vs. SDF+KI was noted between the chemical and biofilm models. While both were equally and more effective than all other interventions in preventing 
enamel demineralization in the chemical model, this was not the case in the biofilm model. Here, KI impaired the efficacy of SDF. These results were in agreement with previous studies on dentin $[9,21,22]$. There are several possible explanations for the present observations:

1) KI may reduce silver ion bioavailability, thus the silver ions are not able to bind with and kill bacteria (anti-bacterial effect of silver). Silver ions are assumed to be primarily responsible for the antimicrobial action of SDF by inhibiting the growth of $S$. mutans, a primary pathogen in dental caries. Thus $S$. mutans is less able to form a biofilm on teeth treated with SDF ex vivo [18-20]. While excess silver ions are removed by $\mathrm{KI}$ in both demineralization models, the impact in the chemical model is negligible as silver ions do not appear to interact in de- and remineralization processes [23].

2) One hypothesis for this data is that $\mathrm{KI}$ increases the organic acid production of bacteria, which in turn causes increased demineralization of tooth structures.

3) Another hypothesis is that the combination of SDF+KI may promote bacterial enzymes involved in carbohydrate metabolism and sugar uptake.

However, the results of a biofilm study employing dentin specimens was in agreement with the results of the chemical model of this study in that SDF+KI was as effective as SDF alone against dental caries [24]. In that study optical density was used to determine the level of bacterial growth, and no data were provided to show correlations between optical density readings and concentrations of $S$. mutans in the solution. To the authors' knowledge no biofilm studies on SDF+KI have been conducted on sound enamel which highlights the novelty of this research.

In both models, SDF and SDF+KI were superior in their ability to prevent caries lesion formation than $\mathrm{AgNO}_{3}$ and DIW. SDF was more effective than KF in both biofilm and chemical models; however, this difference was not significant in the biofilm model. This discrepancy between models can be due to a host of reasons including the interaction of the biofilm with the enamel surface, different degrees of attachment of the biofilm, biofilm growth and acid production. Consequently, this leads to a $\mathrm{pH}$ gradient within the biofilm, which is not comparable to how demineralization occurred in the chemical model. Moreover, in the biofilm model media and plates were changed daily which may cause the biofilm and/or some of the treatments (SDF and KF) to be removed in some group/specimens more than others during transfer.

Topical application of $\mathrm{AgNO}_{3}$ solution had little to no effect in both models and there was no difference between $\mathrm{AgNO}_{3}$ and DIW in both models. It has been shown previously that $\mathrm{AgNO}_{3}$ is washed away if it is applied without a protective layer of fluoride varnish [25].

Based on the VHN results of the chemical model, SDF inhibits demineralization more effectively than KF and $\mathrm{AgNO}_{3}$ alone. Accordingly, it can be assumed that synergistic effects between silver and fluoride exist. However, this assumption was not supported by the biofilm model results as there was no difference between SDF and KF as discussed earlier. 
The TMR data for the chemical model were in agreement with the VHN data. However, this was not the case for the biofilm model. While TMR is considered the gold standard technique for quantifying (changes in) mineral loss and lesion depth of caries lesions [26], it does lack sensitivity in accurately assessing the mineral status of early lesions. Due to the lesser overall extent of demineralization in the biofilm in comparison to the chemical model, the present findings highlight the need to employ several, complementary analytical techniques.

SDF was shown to prevent multi-species cariogenic biofilm growth. The biofilm data (Fig. 4) indicated that growth inhibition of $S$. mutans and other salivary bacteria was higher with SDF alone than with SDF + KI which supports the VHN data. However, $\mathrm{AgNO}_{3}$ did not provide antimicrobial benefits. Destruction of the outer bacterial cell membrane and cytoplasmic extrusion is due to the high reactivity of silver ions to the bacterial enzymes that contain sulfur and phosphorus components in the bacterial cell wall, including the phosphoenolpyruvate phosphotransferase system, which passages sugars through the cell membrane. This high reactivity is due to the difference in charges between the negatively charged bacterial cell wall and the positively charged silver ions which result in an electrostatic adhesion between the bacterial enzymes and the silver particles. Electrostatic adhesion of silver ions with bacterial enzymes inactivates them and prevents metabolic activities of the bacterial enzymes via silver-induced protein coagulation [21,27]. In addition to the effect of ionic silver, fluoride which is the other component of SDF, is the most effective and widely used anti-caries agent found in both self- and professional products. Primarily, fluoride decreases the rate of enamel demineralization and enhances remineralization of enamel caries lesions, which is the main mode of action of fluoride [28]. Fluoride inhibits demineralization by being absorbed onto the hydroxyapatite crystals on the tooth surface. Fluoride also promotes remineralization of tooth mineral hydroxyapatite, and by incorporation of fluoride into the remineralized structure, it thus makes it more resilient to a repeated acid attack $[29,30]$. Furthermore, fluoride has also been shown to prevent the formation of cariogenic biofilms, via binding to bacterial cellular components and influence enzymes which effectively prevent the carbohydrate metabolism of acidogenic oral bacteria and their sugar uptake [30]. Presently, however, there was only a mild antimicrobial fluoride effect as KF did not prevent biofilm growth as effectively as SDF (Fig. 4).

The color measurements utilizing a spectrophotometer were performed to determine changes in the CIE Lab color space of the enamel specimens. The biggest disadvantage of the use of SDF is the dark staining of the tooth surface. Consequently, $\mathrm{Ag}_{3} \mathrm{PO}_{4}, \mathrm{AgO}_{2}$ and $\mathrm{AgS}_{2}$ compounds, found in SDF-treated carious lesions, turn $\mathrm{Ag}^{+}$to metallic silver nanoparticles which after light exposure causes the carious lesions to turn black. This may impact SDF acceptance as a treatment option [4,10,31]. Our results demonstrated that KI helped reverse dark staining caused by SDF in the chemical model immediately after application. This was in agreement with the outcomes of other studies performed on dentin [32,33]. However, KI was not 
able to permanently prevent SDF-related staining after demineralization (Fig 5), which was also observed on dentin previously [34]. Inability of KI in completely removing the discoloration caused by SDF may be due to high photosensitivity of $\mathrm{AgI}$ which can dissociate into metallic silver and iodine by exposure to light. Likewise, there may have been an insufficient amount of KI which led to an excess of free $\mathrm{Ag}^{+}$[34]. Not surprisingly, the DIW group exhibited significantly more whiteness than all other groups in the chemical model due to the formation of an early white spot lesion.

Several limitations need to be highlighted. This laboratory model did not include remineralization periods. The effects of SDF and SDF+KI under chemical and/or bacterial $\mathrm{pH}$-cycling models should be conducted to better understand the efficacy of SDF compared to SDF+KI and its individual components. Furthermore, SDF was only applied once in this study. It has been shown that a single application of SDF is inadequate for constant caries inhibition effects especially on sound enamel. Biannual application for the duration of two years has been recommended to increase the chance of sustained caries arrest, and it may be the same for to prevent new lesions [35]. Moreover, only the immediate effect of SDF was studied presently but not its ability to longitudinally prevent caries. It is noteworthy to mention that the single species $S$. mutans along with multi-species bacteria from human saliva were used. While this is somewhat removed from the complexity of oral biofilms, the key advantage of this biofilm model was that the bacterial cell growth was reliable and comparable among the different treatment groups along with the diversity in the bacterial species [36-38]. Lastly, based on the results of this study it is recommended to analyze lactic acid production in future studies to verify whether applying KI can cause an increase in acid production or not [39].

Within the limitations of the study, SDF may offer an alternative biological approach in preventing primary coronal caries in the future. KI application after SDF significantly improved the dark staining and helped enhance the esthetic outcome by stain reduction. The results from the chemical model show that KI application did not impair the anti-caries efficacy of SDF. However, in the biofilm model, KI diminished the anti-caries efficacy of SDF. Further studies are granted to corroborate whether these effects are sustained using clinical models.

\section{Conclusion}

Under the conditions of this study, SDF appears to be an effective antibacterial and anti-caries topical agent that has the potential to prevent enamel caries. While KI application immediately after SDF treatment can substantially reduce the discoloration caused by SDF, KI impairs SDF's ability to prevent biofilm-mediated demineralization. Further research using clinical models would be needed to establish conclusive evidence.

\section{Declaration of Interest}


The authors declare no conflict of interest.

\section{Ethical approval}

No ethical approval was required prior to the conduct of the present study.

\section{References}

[1] B.H. Oliveira, A. Rajendra, A. Veitz-Keenan, R. Niederman, The effect of silver diamine fluoride in preventing caries in the primary dentition: A systematic review and meta-analysis, Caries Res. 53 (2019) 24-32. https://doi.org/10.1159/000488686.

[2] J. Blackburn, M.A. Morrisey, B. Sen, Outcomes associated with early preventive dental care among Medicaid-enrolled children in Alabama, JAMA Pediatr. 171 (2017) 335-341. https://doi.org/10.1001/jamapediatrics.2016.4514.

[3] J.A Horst, H. Ellenikiotis, P.L. Milgrom, UCSF Protocol for Caries Arrest Using Silver Diamine Fluoride: Rationale, Indications and Consent, J Calif Dent Assoc. 44 (2016) 16-28. https://www.ncbi.nlm.nih.gov/pmc/articles/PMC4778976/

[4] Y.O. Crystal, R. Niederman, Evidence-Based Dentistry Update on Silver Diamine Fluoride, Dent. Clin. North Am. 63 (2019) 45-68. https://doi.org/10.1016/j.cden.2018.08.011.

[5] B. Johhnson, N. Serban, P.M. Griffin, S.L. Tomar, Projecting the economic impact of silver diamine fluoride on caries treatment expenditures and outcomes in young U.S. children, J. Public Health Dent. 79 (2019) 215-221. https://doi.org/10.1111/jphd.12312.

[6] S.S. Gao, S. Zhang, M.L. Mei, E.C.M. Lo, C.H. Chu, Caries remineralisation and arresting effect in children by professionally applied fluoride treatment - a systematic review, BMC Oral Health. 16 (2016) 12. https://doi.org/10.1186/s12903-016-0171-6.

[7] J.C. Llodra, A. Rodriguez, B. Ferrer, V. Menardia, T. Ramos, M. Morato, Efficacy of silver diamine fluoride for caries reduction in primary teeth and first permanent molars of schoolchildren: $36-$ Month clinical trial, J. Dent. Res. 84 (2005) 721-724.

https://doi.org/10.1177/154405910508400807.

[8] I.S. Zhao, S.S. Gao, N. Hiraishi, M.F. Burrow, D. Duangthip, M.L. Mei, E.C.M. Lo, C.H. Chu, Mechanisms of silver diamine fluoride on arresting caries: a literature review, Int. Dent. J. 68 (2018) 67-76. https://doi.org/10.1111/idj.12320.

[9] M.L. Mei, Q.L. Li, C.H. Chu, E.C.M. Lo, L.P. Samaranayake, Antibacterial effects of silver diamine fluoride on multi-species cariogenic biofilm on caries, Ann. Clin. Microbiol. Antimicrob. 12 (2013) 4-4. https://doi.org/10.1186/1476-0711-12-4.

[10] Y.O. Crystal, M.N. Janal, D.S. Hamilton, R. Niederman, Parental perceptions and acceptance of silver diamine fluoride staining, J. Am. Dent. Assoc. 43 (2017) 155-160. https://doi.org/10.1016/j.adaj.2017.03.013.

[11] M. Karched, D. Ali, H. Ngo, In vivo antimicrobial activity of silver diammine fluoride on carious lesions in dentin, J. Oral Sci. 61 (2019) 19-24. https://doi.org/10.2334/josnusd.17-0366.

[12] G.M. Knight, J.M. Mclntyre, Mulyani, The effect of silver fluoride and potassium iodide on the bond strength of auto cure glass ionomer cement to dentine, Aust. Dent. J. 51 (2006) 42-45. https://doi.org/10.1111/j.1834-7819.2006.tb00399.x.

[13] M.A. Hueb De Menezes Oliveira, C.P. Torres, J.M. Gomes-Silva, M.A. Chinelatti, F.C. Hueb De Menezes, R.G. Palma-Dibb, M.C. Borsatto, Microstructure and mineral composition of dental 
enamel of permanent and deciduous teeth, Microsc. Res. Tech. 73 (2010) 572-577.

https://doi.org/10.1002/jemt.20796.

[14] P.R. Wilson, A.D. Beynon, Mineralization differences between human deciduous and permanent enamel measured by quantitative microradiography, Arch. Oral Biol. 34 (1989) 85-88. https://doi.org/10.1016/0003-9969(89)90130-1.

[15] L.J. Wang, R. Tang, T. Bonstein, P. Bush, G.H. Nancollas, Enamel demineralization in primary and permanent teeth, J. Dent. Res. 85 (2006) 359-363.

https://doi.org/10.1177/154405910608500415.

[16] F. Lippert, D. Churchley, R.J. Lynch, Effect of lesion baseline severity and mineral distribution on remineralization and progression of human and bovine dentin caries lesions, Caries Res. 49 (2015) 467-476. https://doi.org/10.1159/000431039.

[17] S. Alshara, F. Lippert, G.J. Eckert, A.T. Hara, Effectiveness and mode of action of whitening dentifrices on enamel extrinsic stains, Clin. Oral Investig. 18 (2014) 563-569.

https://doi.org/10.1007/s00784-013-0981-8.

[18] D.J. White, Reactivity of fluoride dentifrices with artificial caries $i$. Effects on early lesions: $F$ uptake, surface hardening and remineralization, Caries Res. 22 (1987) 27-36. https://doi.org/10.1159/000261013.

[19] H.M. Ayoub, R.L. Gregory, Q. Tang, F. Lippert, Influence of salivary conditioning and sucrose concentration on biofilm-mediated enamel demineralization, J. Appl. Oral Sci. 28 (2020). https://doi.org/10.1590/1678-7757-2019-0501.

[20] Z. M., H. L.B., E. R.A., C. L., L. J.Y., T.C. J.M., C. W., Biofilm layers affect the treatment outcomes of NaF and Nano-hydroxyapatite, J. Dent. Res. 94 (2015) 602-607.

[21] H.H. Hamama, C.K. Yiu, M.F. Burrow, Effect of silver diamine fluoride and potassium iodide on residual bacteria in dentinal tubules, Aust. Dent. J. 60 (2015) 80-87. https://doi.org/10.1111/adj.12276.

[22] G.M. Knight, J.M. McIntyre, G.G. Craig, Mulyani, P.S. Zilm, N.J. Gully, Inability to form a biofilm of Streptococcus mutans on silver fluoride- and potassium iodide-treated demineralized dentin, Quintessence Int. 40 (2009) 155-161.

[23] O.Y. Yu, I.S. Zhao, M.L. Mei, E.C.M. Lo, C.H. Chu, Effect of silver nitrate and sodium fluoride with tri-calcium phosphate on streptococcus mutans and demineralised dentine, Int. J. Mol. Sci. 19 (2018). https://doi.org/10.3390/ijms19051288.

[24] K. G.M., M. J.M., C. G.G., Mulyani, Z. P.S., G. N.J., An in vitro model to measure the effect of a silver fluoride and potassium iodide treatment on the permeability of demineralized dentine to Streptococcus mutans, Aust. Dent. J. 50 (2005) 242-245.

[25] I.S. Zhao, M.L. Mei, Q.L. Li, E.C.M. Lo, C.H. Chu, Arresting simulated dentine caries with adjunctive application of silver nitrate solution and sodium fluoride varnish: an in vitro study, Int. Dent. J. 67 (2017) 206-214. https://doi.org/10.1111/idj.12291.

[26] J.J. Ten Bosch, B. Angmar-Mansson, A review of quantitative methods for studies of mineral content of intra-oral caries lesions, J Dent Res. 70 (1991) 2-14.

[27] T. Ishiguro, G. Mayanagi, M. Azumi, H. Otani, A. Fukushima, K. Sasaki, N. Takahashi, Sodium fluoride and silver diamine fluoride-coated tooth surfaces inhibit bacterial acid production at the bacteria/tooth interface, J. Dent. 84 (2019) 30-35. https://doi.org/10.1016/j.jdent.2018.12.017.

[28] F. Lippert, E.E. Newby, R.J.M. Lynch, V.K. Chauhan, B.R. Schemehorn, Laboratory assessment of the anticaries potential of a new dentifrice, J. Clin. Dent. 20 (2009) 45-49.

[29] Y.O. Crystal, A.A. Marghalani, S.D. Ureles, J.T. Wright, R. Sulyanto, K. Divaris, M. Fontana, L. Graham, Use of silver diamine fluoride for dental caries management in children and adolescents, including those with special health care needs, Pediatr. Dent. 39 (2017) 135-145. 
[30] M.L. Mei, Q.L. Li, C.H. Chu, E.C.M. Lo, L.P. Samaranayake, Antibacterial effects of silver diamine fluoride on multi-species cariogenic biofilm on caries, Ann. Clin. Microbiol. Antimicrob. 12 (2013)

4. https://doi.org/10.1186/1476-0711-12-4.

[31] Y. Li, Y. Liu, W.J. Psoter, O.M. Nguyen, T.G. Bromage, M.A. Walters, B. Hu, S. Rabieh, F.C. Kumararaja, Assessment of the Silver Penetration and Distribution in Carious Lesions of Deciduous Teeth Treated with Silver Diamine Fluoride, Caries Res. 53 (2019) 431-440. https://doi.org/10.1159/000496210.

[32] I.S. Zhao, S. Chu, O.Y. Yu, M.L. Mei, C.H. Chu, E.C.M. Lo, Effect of silver diamine fluoride and potassium iodide on shear bond strength of glass ionomer cements to caries-affected dentine, Int. Dent. J. 69 (2019) 341-347. https://doi.org/10.1111/idj.12478.

[33] J. Gupta, M. Thomas, M. Radhakrishna, N. Srikant, K. Ginjupalli, Effect of silver diamine fluoridepotassium iodide and $2 \%$ chlorhexidine gluconate cavity cleansers on the bond strength and microleakage of resin-modified glass ionomer cement, J. Conserv. Dent. 22 (2019) 201-206. https://doi.org/10.4103/JCD.JCD 48518.

[34] I.S. Zhao, M.L. Mei, M.F. Burrow, E.C.M. Lo, C.H. Chu, Effect of silver diamine fluoride and potassium iodide treatment on secondary caries prevention and tooth discolouration in cervical glass ionomer cement restoration, Int. J. Mol. Sci. 18 (2017) 340. https://doi.org/10.3390/ijms18020340.

[35] Y.O. Crystal, S. Rabieh, M.N. Janal, S. Rasamimari, T.G. Bromage, Silver and fluoride content and short-term stability of 38\% silver diamine fluoride, J. Am. Dent. Assoc. 150 (2019) 140-146. https://doi.org/10.1016/j.adaj.2018.10.016.

[36] O.Y. Yu, I.S. Zhao, M.L. Mei, E.C.M. Lo, C.H. Chu, Caries-arresting effects of silver diamine fluoride and sodium fluoride on dentine caries lesions, J. Dent. 78 (2018) 65-71. https://doi.org/10.1016/j.jdent.2018.08.007.

[37] O.Y. Yu, M.L. Mei, I.S. Zhao, Q.L. Li, E.C.M. Lo, C.H. Chu, Remineralisation of enamel with silver diamine fluoride and sodium fluoride, Dent. Mater. 34 (2018) e344-e352.

https://doi.org/10.1016/j.dental.2018.10.007.

[38] S. Savas, E. Kucukyılmaz, E.U. Celik, M. Ates, Effects of different antibacterial agents on enamel in a biofilm caries model, J. Oral Sci. 57 (2015) 367-372. https://doi.org/10.2334/josnusd.57.367.

[39] B.Y. Liu, E.C.M. Lo, C.M.T. Li, Effect of silver and fluoride ions on enamel demineralization: A quantitative study using micro-computed tomography, Aust. Dent. J. 57 (2012) 65-70. https://doi.org/10.1111/j.1834-7819.2011.01641.x. 


\section{Figure legends}

Fig. 1. Schematic of the experimental procedures.

Fig. 2. Chemical model - percent change in surface microhardness $(\% \mathrm{SMH}$ change; mean \pm standard deviation) from baseline. Different letters highlight statistically significant differences between treatment groups.

Fig. 3. Biofilm model - percent change in surface microhardness (\%SMH change; mean \pm standard deviation) from baseline. Different letters highlight statistically significant differences between treatment groups.

Fig. 4. Biofilm model - changes in colony-forming units (Log CFU/ml; mean \pm standard deviation) from baseline. Different letters highlight statistically significant differences between treatment groups.

Fig. 5. Chemical model - color change ( $\Delta \mathrm{L}^{*}$; mean \pm standard deviation). Black bars: mean change between after treatment and baseline-lower case letter for black bars shows after treatment change from baseline; gray bars: mean change between post intervention and baseline-upper case letter for gray bars shows postintervention change from baseline. Different letters highlight statistically significant differences between treatment groups.

Fig. 6. Biofilm model - color change ( $\Delta \mathrm{L}^{*}$; mean \pm standard deviation). Black bars: mean change between post intervention and baseline. Different letters highlight statistically significant differences between treatment groups. 
Table 1 - TMR data (all means \pm standard deviations) for both models

\begin{tabular}{|c|c|c|c|c|}
\hline & Chemical model & & Biofilm model & \\
\hline Intervention & $\Delta \mathrm{Z}(\mathrm{vol} \% \min \times \mu \mathrm{m})$ & $\mathrm{L}(\mu \mathrm{m})$ & $\Delta \mathrm{Z}(\mathrm{vol} \% \min \times \mu \mathrm{m})$ & $\mathrm{L}(\mu \mathrm{m})$ \\
\hline SDF & $96 \pm 94$ & $6 \pm 7$ & $279 \pm 175$ & $16 \pm 14$ \\
\hline $\mathrm{SDF}+\mathrm{KI}$ & $124 \pm 99$ & $6 \pm 7$ & $323 \pm 221$ & $12 \pm 11$ \\
\hline $\mathrm{KF}$ & $198 \pm 210$ & $9 \pm 10$ & $218 \pm 228$ & $12 \pm 13$ \\
\hline $\mathrm{AgNO}_{3}$ & $1390 \pm 497$ & $49 \pm 13$ & $343 \pm 166$ & $17 \pm 8$ \\
\hline DIW & $1334 \pm 478$ & $52 \pm 12$ & $407 \pm 181$ & $20 \pm 8$ \\
\hline
\end{tabular}




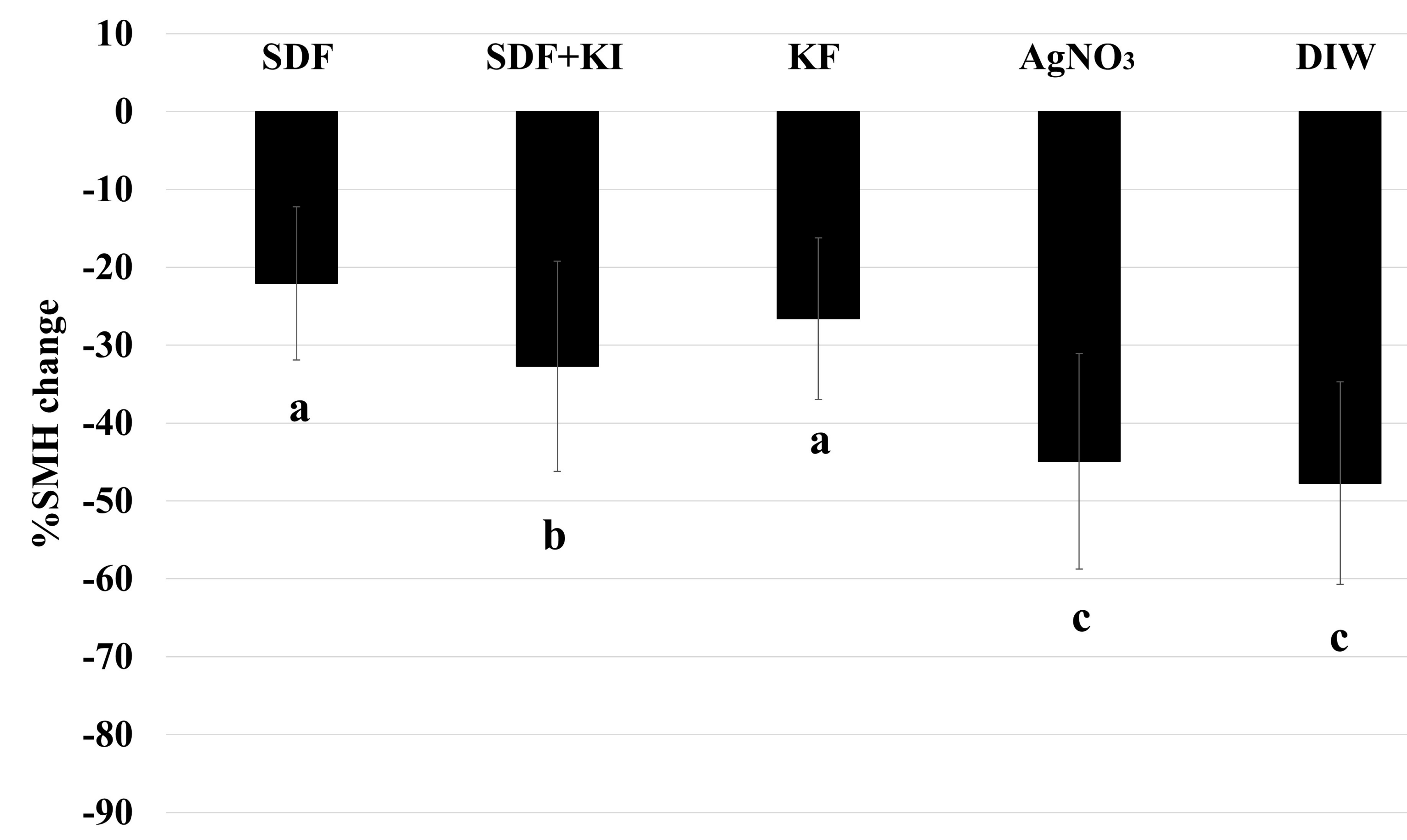




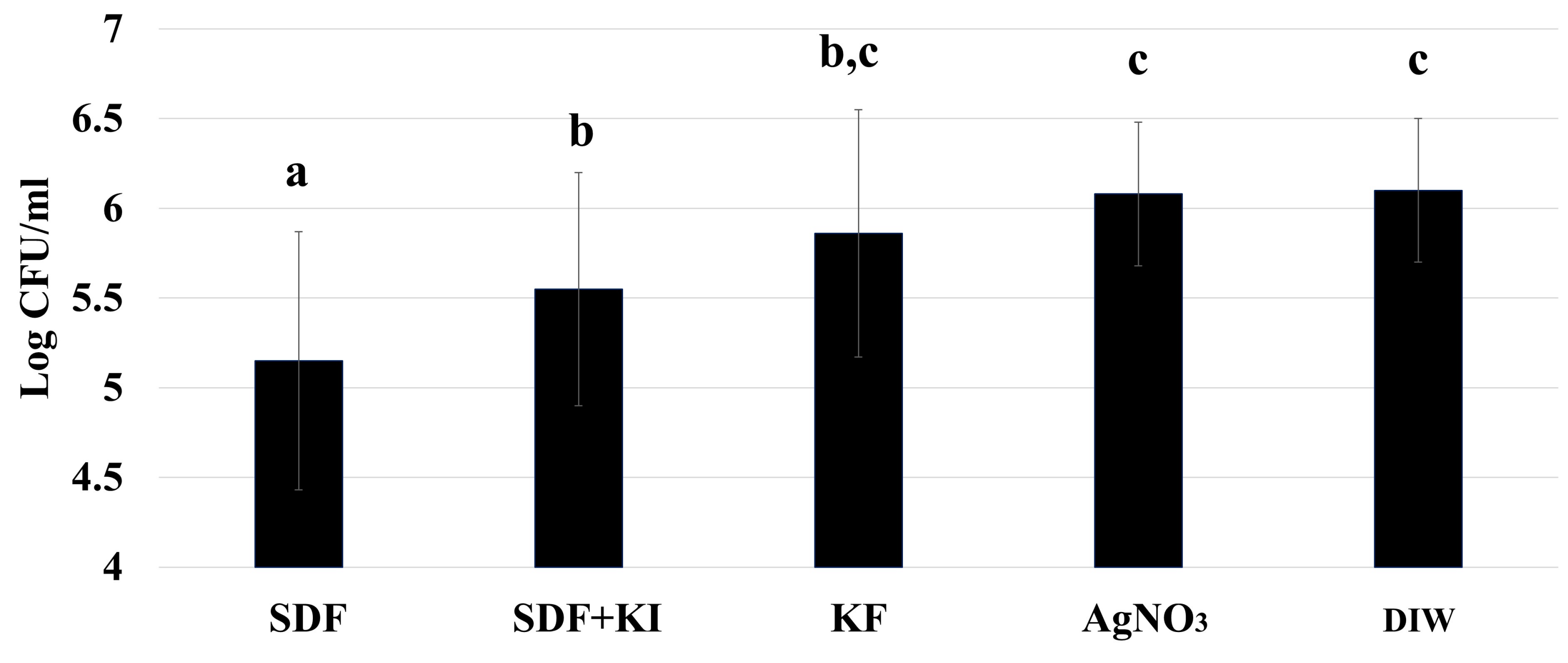


$15 \quad$ SDF

10

$\begin{array}{ll}* & 0 \\ \gtrless & -5\end{array}$

$-10$

$\mathbf{a}$

A
KF

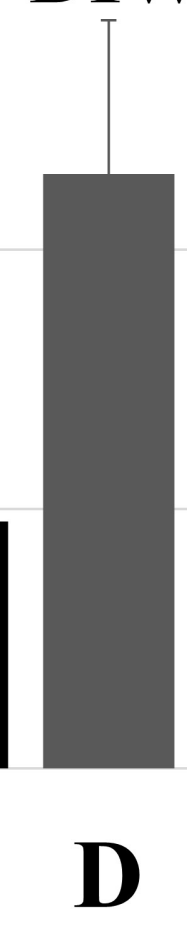

$-15$

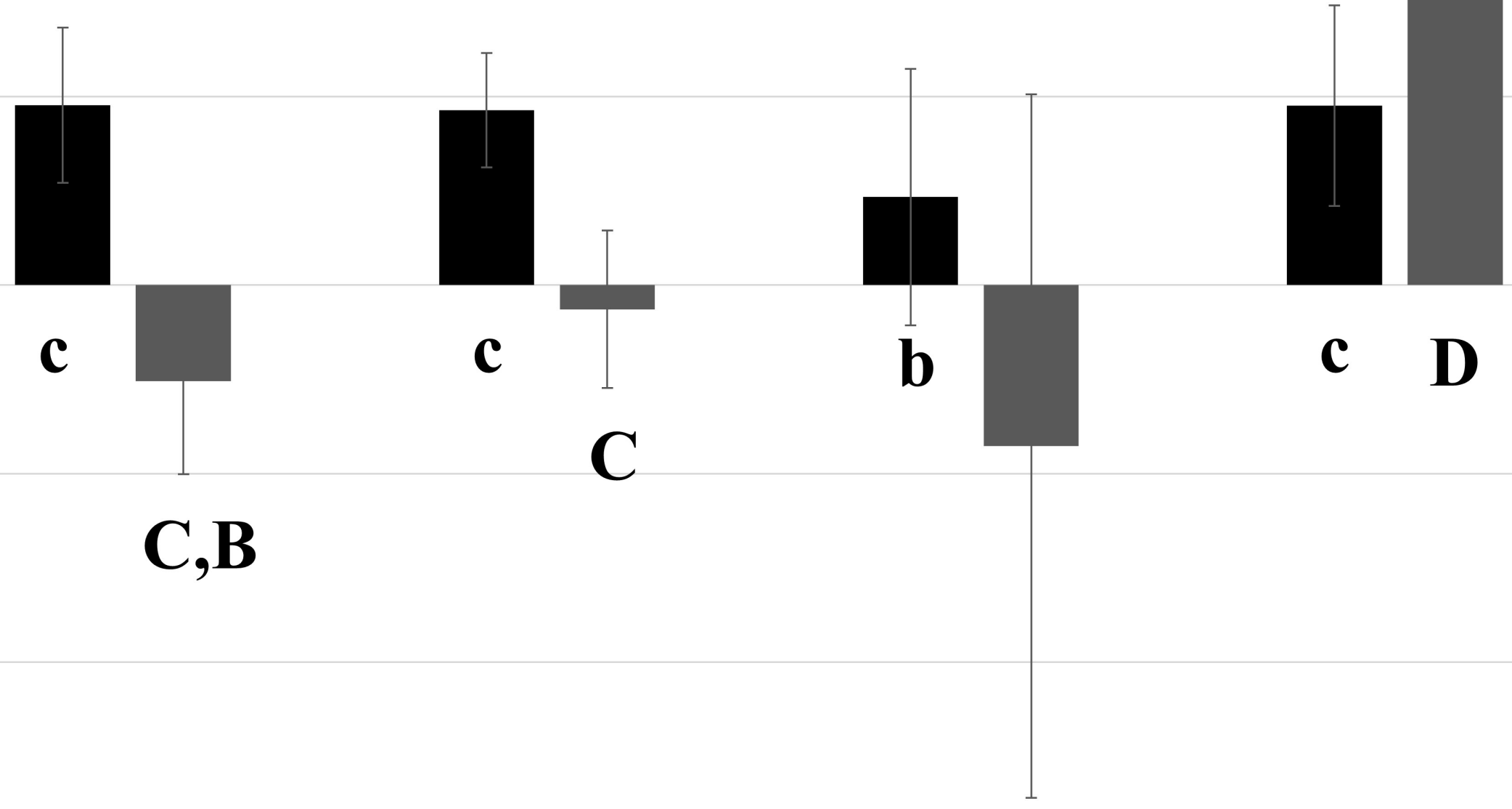

A,B 
